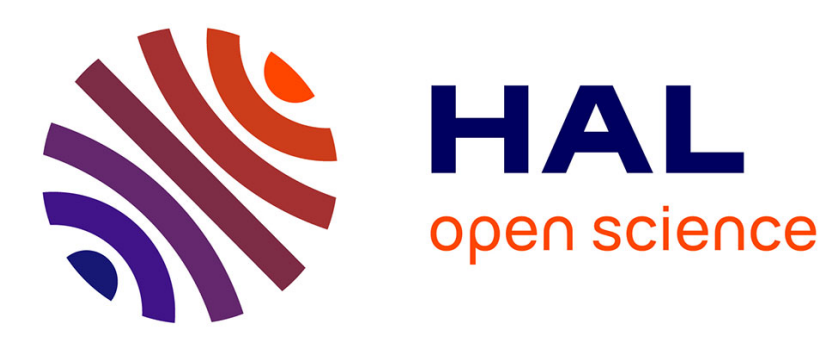

\title{
Fabry-Pérot-Michelson interferometer using higher-order Laguerre-Gauss modes
}

\author{
A. Gatto, M. Tacca, F. Kéfélian, Christelle Lesvigne-Buy, M. Barsuglia
}

\section{To cite this version:}

A. Gatto, M. Tacca, F. Kéfélian, Christelle Lesvigne-Buy, M. Barsuglia. Fabry-Pérot-Michelson interferometer using higher-order Laguerre-Gauss modes. Physical Review D, 2014, 90 (12), pp.122011. 10.1103/PhysRevD.90.122011 . hal-01101371

\section{HAL Id: hal-01101371 \\ https://hal.science/hal-01101371}

Submitted on 30 Mar 2015

HAL is a multi-disciplinary open access archive for the deposit and dissemination of scientific research documents, whether they are published or not. The documents may come from teaching and research institutions in France or abroad, or from public or private research centers.
L'archive ouverte pluridisciplinaire HAL, est destinée au dépôt et à la diffusion de documents scientifiques de niveau recherche, publiés ou non, émanant des établissements d'enseignement et de recherche français ou étrangers, des laboratoires publics ou privés. 


\title{
Fabry-Pérot-Michelson interferometer using higher-order Laguerre-Gauss modes
}

\author{
A. Gatto, M. Tacca, F. Kéfélian, C. Buy, and M. Barsuglia \\ Laboratoire AstroParticule et Cosmologie (APC), Université Paris Diderot, CNRS/IN2P3, CEA/Irfu, \\ Observatoire de Paris, Sorbonne Paris Cité, 10, rue Alice Domon et Léonie Duquet, 75013 Paris, France
}

(Received 9 October 2014; published 31 December 2014)

\begin{abstract}
One of the main noise sources for future interferometric gravitational wave detectors will be the thermal fluctuations of the mirrors used as test masses. To reduce the effect of this noise without changing the mirror material, size and temperature, the use of Laguerre-Gauss (LG) modes has been proposed. In this work we report the first experimental results obtained with a Fabry-Pérot Michelson interferometer operated with Laguerre-Gauss $\mathrm{LG}_{33}$ as input mode and developed as a table-top prototype to test alignment and matching procedures, possible control issues and optical performances of the $\mathrm{LG}_{33}$. Among the results obtained, we have demonstrated the degradation of the interferometer contrast of the $\mathrm{LG}_{33}$ with respect to the Gaussian beam, predicted by several previous simulations works and due to the degeneracy of LG modes having the same order. In addition, the observed contrast degradation for the $\mathrm{LG}_{33}$ as well as the interferometer optical properties have been explained using a numerical simulation.
\end{abstract}

DOI: 10.1103/PhysRevD.90.122011

PACS numbers: 04.80.Nn, 95.55.Ym, 95.75.Kk

\section{INTRODUCTION}

Gravitational waves $(\mathrm{GW})$ are propagating perturbations in the space-time metric predicted by Einstein's general relativity theory. Current attempts to detect GW are mainly based on kilometer-scale Michelson optical interferometers. In the past decade, first generation $\mathrm{GW}$ detectors such as GEO [1], LIGO [2] and Virgo [3] have completed several observational runs, but no detection has been made because of the expected low rate of astrophysical events [4]. Second generation GW detectors [5-8] are now under construction, and their sensitivity will be limited in their central frequency band by Brownian thermal noise of the coatings of the mirrors used as test masses. Consequently, for any substantial improvement of the sensitivity of future interferometric gravitational-wave detectors, the reduction of the impact of this noise source will be necessary. One option is to use the same mirror material and size, but a laser beam with an intensity profile wider or flatter than the fundamental Gaussian mode [9]. This should reduce the effect of mirror thermal noise on the optical beam phase thanks to a higher spatial averaging on the mirror surface. Among the possible beam profiles, higher-order LaguerreGauss (LG) modes [10,11] present the advantage of having spherical wave fronts and, therefore, of being compatible with spherical mirrors, a well established technology.

The Laguerre-Gauss modes form a complete and orthogonal set by which it is possible to describe the shape of every possible paraxial beam. A generic helical $\mathrm{LG}_{p, l}$ mode is defined by Eq. (1) described in [12,13], where $k$ is the wave number, $\Psi(z)$ is the Gouy phase, $L_{p}^{|l|}(x)$ is the generalized Laguerre polynomial, $w(z)$ and $R_{c}(z)$ are the size and radius of curvature of the beam at position $z$. Any Laguerre-Gauss mode is identified by two indices, the radial index $p$ and the azimuthal index $l$. An $\mathrm{LG}_{p l}$ beam with $l \neq 0$ has $p+1$ radial nodes and $2 \pi \ell$ phase shifts of the helical phase front occurring in one full rotation of the azimuthal angle $\phi$.

$$
\begin{aligned}
\psi_{p, l}(r, \phi, z)= & \frac{1}{w(z)} \sqrt{\frac{2 p !}{\pi(|l|+p) !}}\left(\frac{\sqrt{2} r}{w(z)}\right) \\
& \times L_{p}^{|l|}\left(\frac{2 r^{2}}{w^{2}(z)}\right) \exp (i(2 p+|l|+1) \Psi(z)) \\
& \times \exp \left(-\frac{k r^{2}}{2 R_{c}(z)}-\frac{r^{2}}{w^{2}(z)}+i l \phi\right)
\end{aligned}
$$

Up to now, two table-top experiments have demonstrated the generation of a high purity $\mathrm{LG}_{33}$ mode for $\mathrm{GW}$ detection using a diffractive element, either a liquidcrystal-on-silicon spatial-light modulator [14] or a fusedsilica plate [15], in combination with a linear mode-cleaner cavity. Two further experiments have investigated this technique at scales closer to a real gravitational wave detector by increasing either the laser power or the length of the optical system: the former [16] was focused on the $\mathrm{LG}_{33}$ high-power generation by a diffractive phase plate, while the goal of the latter [17] was to inject an $\mathrm{LG}_{33}$ mode into a suspended 10-m-long Fabry-Pérot cavity in vacuum. In parallel with this experimental activity, a simulation program [18-20] has been crucial to highlight the issues related to the degeneracy of LG modes of the same order. From Eq. (1), modes of the same order $2 p+l$ have the same value of the Gouy phase. As a consequence, modes of the same order are degenerate in resonant cavities. Since any deviation of the mirror surface from the ideal spherical shape will induce a coupling between modes and since the 
degenerate modes will resonate in the cavity together with the injected one, a non-negligible fraction of power will be transferred to degenerate modes. This would lead to a degradation of the interferometer visibility [18-20]. Considering the state-of-the-art mirror surface quality, the degeneracy is presently the main issue for the exploitation of the LG modes in future GW detectors.

In this work we report the first experimental results obtained with a Fabry-Pérot-Michelson interferometer using a Laguerre-Gauss $\mathrm{LG}_{33}$ as the input mode. The interferometer has been developed as a table-top prototype to test alignment and matching procedures, possible control issues and optical performances in a configuration closer to the ones of GW interferometers and complementary to previous experiments. Among the results obtained, we have demonstrated the effects of the degeneracy on the interferometer visibility, and we have demonstrated that the optical performances of the interferometer are in agreement with numerical simulations.

In Sec. II the experimental setup is presented. Then, the main experimental results are reported in Sec. III in terms of interferometer characterization, control and beam shapes. The comparison between experimental data and numerical simulations is described in Sec. IV. Finally, the conclusions and the future steps are presented, respectively, in Secs. V and VI.

\section{EXPERIMENTAL SETUP}

The optical scheme of the experiment is shown in Fig. 1. It can be divided into three main parts: the $\mathrm{LG}_{33}$ generation system (generator), the optics needed to inject the beam to the interferometer (injection system) and the Fabry-PérotMichelson interferometer itself (interferometer). Since the alignment and the matching are easier with the Gaussian beam, in this setup either the $\mathrm{LG}_{33}$ mode or the Gaussian mode can be used.

The quality of the modes in various points has been estimated by using an adimensional intensity overlap integral $\gamma$ (already used in $[15,16]$ ), defined as

$$
\gamma=\frac{\iint I_{\text {meas }} \times I_{\text {theory }} d S}{\sqrt{\iint I_{\text {meas }}^{2} d S} \times \sqrt{\iint I_{\text {theory }}^{2} d S}},
$$

where $I_{\text {meas }}=\left|\psi_{\text {meas }}\right|^{2}$ is the transverse intensity distribution acquired by a beam profiler camera, $I_{\text {theory }}=\left|\psi_{\text {theory }}\right|^{2}$ is the theoretical intensity distribution, calculated from Eq. (1), and dS is the infinitesimal surface element. Since the beam profiler camera acquires only the intensity, in Eq. (2) the phase of the two modes is neglected and, therefore, $\gamma$ is an upper limit of the mode purity of the measured beam.

In the following paragraphs the experimental setup is described with more details.

\section{A. $\mathbf{L G}_{\mathbf{3 3}}$ generator}

The $\mathrm{LG}_{33}$ generator is described in detail in a previous work [15]. It is mainly composed by a diffractive phase plate and a linear mode-cleaner cavity. A Gaussian beam generated by a 500-mW 1064-nm Nd:YAG laser passes through an electro-optical phase modulator (EOM), needed for the generation of the radio-frequency sidebands, then it goes through an optical telescope and through the diffractive phase plate. To enhance the modal purity of the pseudo- $\mathrm{LG}_{33}$ produced by the phase plate, the beam is filtered by a $30-\mathrm{cm}$-long plano-concave cavity of finesse
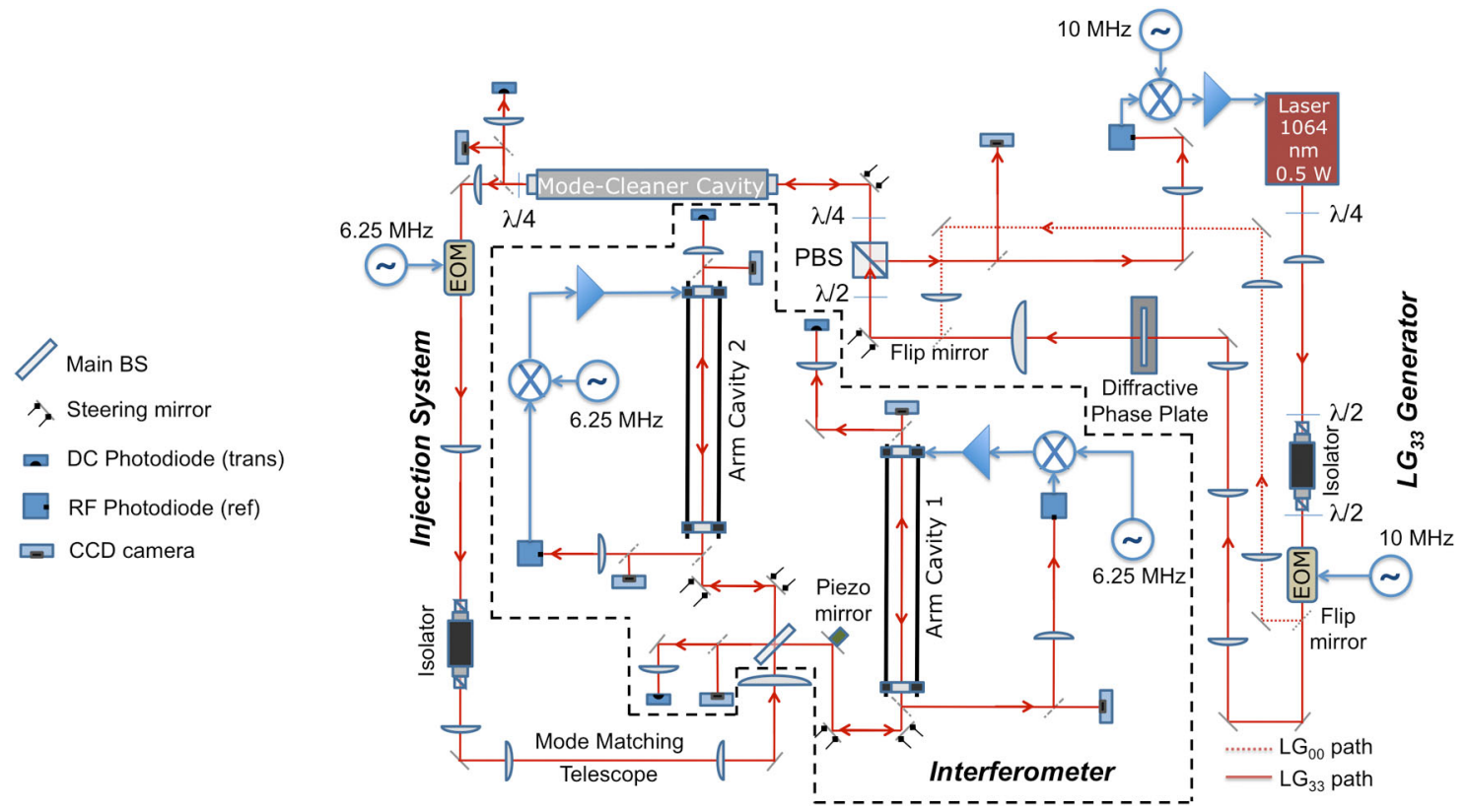

FIG. 1 (color online). Optical scheme of the experimental set-up. 
100 (the mode cleaner). To have the $\mathrm{LG}_{33}$ resonant in the mode cleaner, the frequency of the Nd:YAG laser is locked to the length of the mode-cleaner cavity using the PoundDrever-Hall technique. Compared to [15], the mechanical stability of the mode-cleaner cavity has been improved by adding elastic dampers on the cradle holding the cavity. This allows one to work on the interferometer without unlocking the mode cleaner.

The procedure used for the mode-cleaner initial alignment is similar to the one described in [15], and it has been the following:

(1) The optical axis of the Gaussian and the $\mathrm{LG}_{33}$ beams have been superposed at the level of the two steering mirrors before the mode-cleaner cavity. A very precise superposition has been obtained using two CCD cameras placed in near field and far field.

(2) The Gaussian beam has been aligned on the modecleaner cavity acting on the two steering mirrors. In this way, the $\mathrm{LG}_{33}$ is also aligned at the same time.

(3) The input has been switched from the Gaussian to the $\mathrm{LG}_{33}$ beam.

At the end of this procedure, the $\mathrm{LG}_{33}$ is the dominant mode resonating inside the mode cleaner and only a slight alignment is necessary to maximize its coupling.

In Fig. 2(a) the intensity distribution at the mode-cleaner output is reported, while Fig. 2(b) shows the intensity distribution of a theoretical $\mathrm{LG}_{33}$ beam, calculated by Eq. (1) for a beam at the same distance from the modecleaner waist (set on the flat input mirror). We remark that the spatial high frequency structures visible in the measured distribution are due to the imaging system, and they are present also for the Gaussian beam. In Fig. 3 the cross sections of the beam measured at the mode-cleaner output (blue and green lines for $x$ and $y$ directions, respectively) are compared to the theoretical cross sections at the same distance from the waist. The overlap integral, defined by Eq. (2), is higher than $99 \%$.

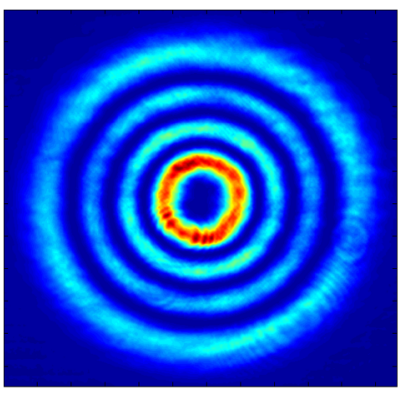

(a)

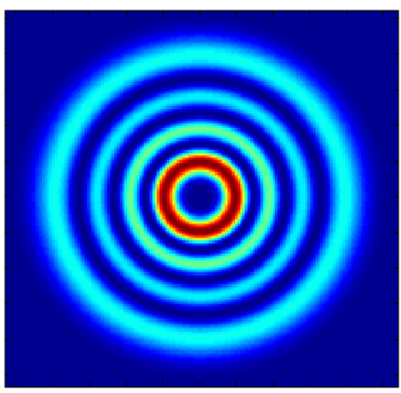

(b)
FIG. 2 (color online). Intensity distribution recorded at the mode-cleaner output (a) and theoretical $\mathrm{LG}_{33}$ distribution at the same distance from waist (b). We remark that the spatial high frequency structures visible in the measured distribution are due to the imaging system and they are also present for the Gaussian beam.

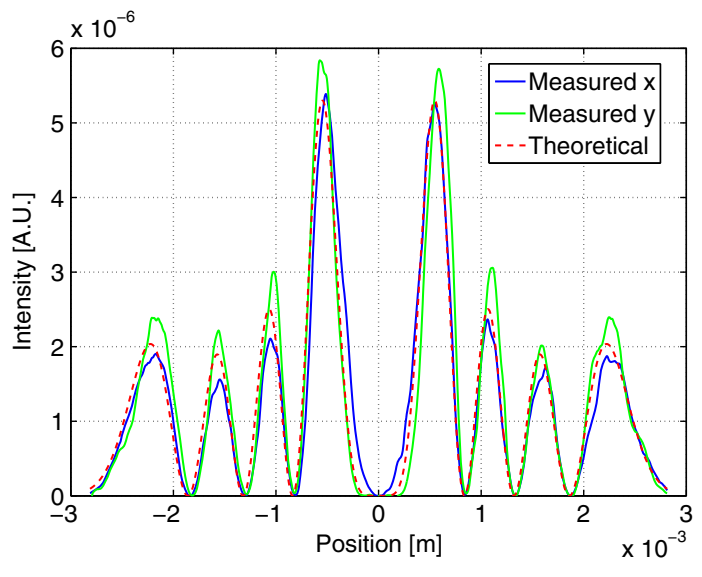

FIG. 3 (color online). Cross sections of the beam measured at the mode-cleaner output (blue and green lines for $x$ and $y$ directions, respectively) and theoretical cross section (red dotted line) at the same distance from waist.

\section{B. Injection system}

The beam first passes through an electro-optical phase modulator (needed for the generation of the $6.25 \mathrm{MHz}$ radio-frequency sidebands for the lock of the two arm cavities with a Pound-Drever-Hall scheme) and a Faraday isolator (needed for the rejection of the reflected light from the interferometer which can unlock the mode cleaner and increase the noise of the interferometer). To keep the clipping losses lower than $1 \%$ passing through these components, the radius of the $\mathrm{LG}_{33}$ mode has to be more than 2 times smaller than the radius of the Gaussian mode, which means a reduction of the Rayleigh range by $\sim 5$. For this reason, great care has been taken in the choice of the focusing lenses and in the alignment of the EOM and the Faraday isolator, in order to avoid any clipping loss or degradation of the beam through these components (whose apertures are of the order of $\mathrm{mm}$ and lengths of 5-10 cm). Then, a mode matching 4-lenses telescope (allowing a quasi-independent adjustment of the beam waist size and waist position) is used to match the beam parameters with the ones of the Fabry-Pérot cavities.

In Fig. 4 the transverse intensity distributions at the input of the two arm cavities are reported. No degradation of the $\mathrm{LG}_{33}$ beam due to the injection system is observed. The intensity overlap integrals, as defined above, are higher than $99 \%$.

\section{Interferometer}

The interferometer is a Michelson with Fabry-Pérot cavities in the arms. The 30-cm-long cavities are composed of a flat input mirror (ITM) and a 50-cm radius of curvature concave end mirror (ETM). The reflectivities of the input and end mirrors are, respectively, $97 \%$ and $99.9 \%$, corresponding to a finesse of 200 . The mirrors are $1 \mathrm{in}$. diameter made of fused silica with a surface quality of $\lambda / 20$. The end 


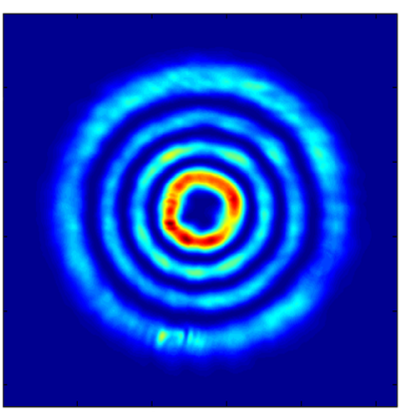

(a)

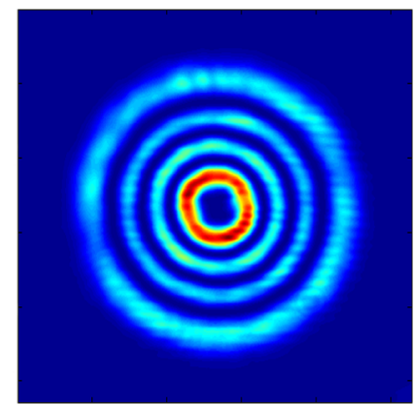

(b)

FIG. 4 (color online). Intensity patterns recorded at arm cavities' inputs. (a) Cavity 1, (b) cavity 2.

mirror mounts are provided with piezoactuators in order to control the cavities' length. The spot size is $288 \mu \mathrm{m}$ and $455 \mu \mathrm{m}$, respectively, on the input and end mirrors. The reflected and transmitted beams from the cavities and the beam at the interferometer output are detected by photodetectors and CCD cameras. The two photodiodes receiving the reflected beams are also demodulated at $6.25 \mathrm{MHz}$ in order to generate error signals to control the cavity lengths.

To keep the interferometer on its working point, three independent lengths have to be controlled: the length of the two Fabry-Pérot cavities (in order to have the cavities in resonance with the input laser light) and the arm-length difference of the Michelson. The control of the armcavities' lengths has been performed by the extraction of Pound-Drever-Hall error signals and, after filtering, by applying corrections to the end mirrors through the piezoelectric actuators (PZT) actuators. The error signal for the control of the Michelson arm-length difference has been generated by a dithering technique: the Michelson length is modulated at $1 \mathrm{kHz}$ by a mirror with a PZT actuator installed in one of the Michelson arms and the corresponding signal is detected at the interferometer output. Corrections are then sent at the same PZT actuator.

For the three lengths, the signals are sent to analog-todigital converters working at $20 \mathrm{kHz}$ and digitized. Digital filters are applied using dedicated hardware and software developed in the framework of the Virgo project [21], and correction signals are sent to each mirror mount after digital-to-analog conversion. An automatic lock acquisition procedure, implemented through a software script, sends a voltage ramp to the PZT actuators to search for the interferometer working point and uses the transmitted powers as a trigger to enable the lock.

\section{INTERFEROMETER: EXPERIMENTAL RESULTS}

\section{A. Interferometer alignment, matching and control}

At the input of the interferometer, the Gaussian beam parameters and the beam axis are the same for the $\mathrm{LG}_{33}$

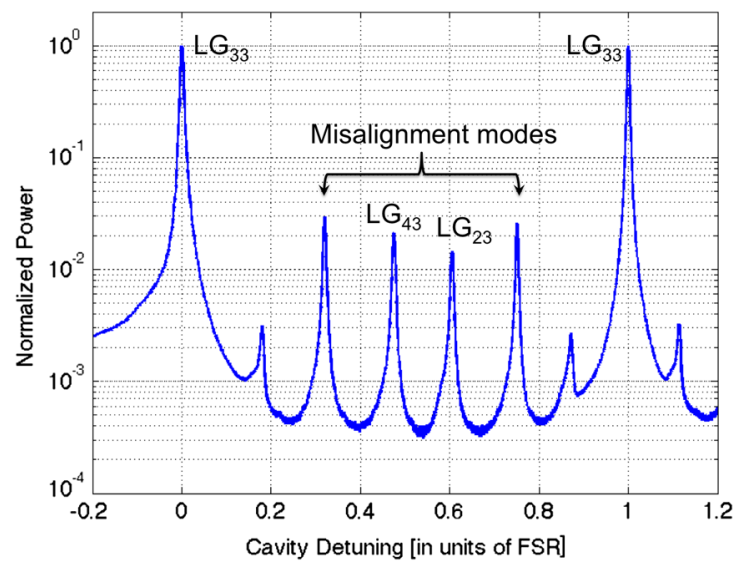

FIG. 5 (color online). Length scan of cavity 1 over a free spectral range with the $\mathrm{LG}_{33}$ as input mode. The structure visible on the two sides of all the resonance peaks, and especially on the $\mathrm{LG}_{33}$ ones, is due to the radio-frequency modulation sidebands. The small peaks at the side of the main resonances are due to a combination of mismatching and misalignments.

and for the Gaussian beams, since they are completely defined by the mode-cleaner cavity. For this reason, the preliminary alignment of the interferometer and the mode matching of the cavities have been performed using the fundamental Gaussian mode, and only a slight optimization of the alignment is required after switching to the $L_{33}$ beam.

In Fig. 5 a length scan for cavity 1 is shown after the switch to the $\mathrm{LG}_{33}$ and the optimization of alignment and matching (similar results are obtained for cavity 2). The power coupled on higher-order modes due to residual misalignments and mismatching is shown in Table I for both the Gaussian and the $\mathrm{LG}_{33}$ beams. We remark that the $\mathrm{LG}_{33}$ sensitivity to mode-matching errors is greater by a factor of $\sim 40$ with respect to the Gaussian beam (which has the same Gaussian parameters) and the sensitivity to misalignments is greater by a factor of $\sim 8$. These ratios are confirmed by a numerical simulation (described later in detail), as visible in Fig. 12 below. The slight widening of the main peaks is due to the radio-frequency sidebands.

TABLE I. Total power coupled in higher-order modes due to residual misalignments and mismatching for Gaussian (top) and $\mathrm{LG}_{33}$ (bottom) input modes. The total coupled power is the sum on all the mismatching or the misalignments higher-order modes.

\begin{tabular}{lcc}
\hline \hline Gaussian & Misalignments & Mismatching \\
\hline Cavity 1 & $0.8 \%$ & $0.0875 \%$ \\
Cavity 2 & $1.6 \%$ & $0.075 \%$ \\
\hline LG $_{33}$ & Misalignments & Mismatching \\
\hline Cavity 1 & $6 \%$ & $3.5 \%$ \\
Cavity 2 & $12 \%$ & $4 \%$ \\
\hline \hline
\end{tabular}




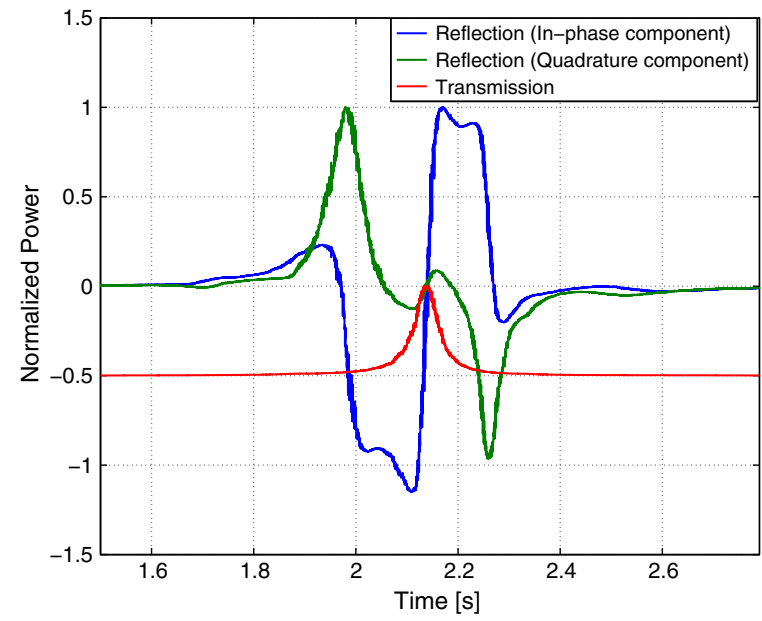

FIG. 6 (color online). Pound-Drever-Hall signals for the $\mathrm{LG}_{33}$ (for cavity 1) near the cavity resonance (blue curve: in-phase component; green curve: quadrature component) superposed to the transmitted power (red curve).

As already verified in other experiments [14,15], the Pound-Drever-Hall error signals of the Fabry-Pérot cavities for the Gaussian and $\mathrm{LG}_{33}$ modes are the same. The error signal for the $\mathrm{LG}_{33}$ mode is shown in Fig. 6. Once the cavities are locked, the error signal for the Michelson length is the one of a simple Michelson with no cavities in the arms, as expected.

The powers transmitted by the two cavities during a typical acquisition of the resonance (using the $\mathrm{LG}_{33}$ ) are shown in Fig. 7(a). Figure 7(b) shows the lock acquisition of the Michelson length (at $t=17 \mathrm{~s}$ ). Before $t=17 \mathrm{~s}$, the Michelson arm-length difference is artificially modulated at a very low frequency. At $t=17 \mathrm{~s}$ a correction signal is applied and the Michelson arm-length difference is then locked on the dark fringe condition (destructive interference). Fluctuations in the transmitted powers of the cavities and in the power at the interferometer output are mainly due to the acoustic noise in the laboratory.

\section{B. Image analysis and interferometer visibility}

The transverse intensity distributions of the beams transmitted by the two cavities are shown in Figs. 8(a)-8(c). We remark a degradation of the beam quality with respect to the input beams, also confirmed by the lower values of the overlap integrals between the measured beams and the theoretical $\mathrm{LG}_{33}$ beams at the same position $(94.1 \%$ for cavity 1 and $95.8 \%$ for cavity 2 ). Both the patterns show an elliptic distribution. As explained later, this is an effect of the degeneracy of the $\mathrm{LG}_{33}$ with modes of the same order, and it is mainly due to the astigmatism of the end mirrors. Since the relative orientation of the astigmatism axes has a huge impact on the interferometer visibility, one of the two end mirrors has been turned around the optical axes, in order to align as much as possible the astigmatism axes of the two cavities (since the operation is manually made, a slight relative rotation between the astigmatism axes of the two arm cavities could not be avoided).

We also remark that the shapes of transmitted beams change by slightly modifying the global alignment of the interferometer (and then the position of the beam impinging on the end mirrors): this suggests that the local astigmatism is not constant.

The intensity distributions for the reflected beams are shown in Figs. 8(b)-8(d). The asymmetry of the distributions is due to the presence of residual misalignments. The overlap integrals for the reflected beams are $66.3 \%$ for cavity 1 and $70.2 \%$ for cavity 2 .

The intensity pattern recorded at the interferometer output when the two cavities are at resonance and the Michelson is on the dark fringe is shown in Fig. 9. The fringe visibility of the interferometer is defined as

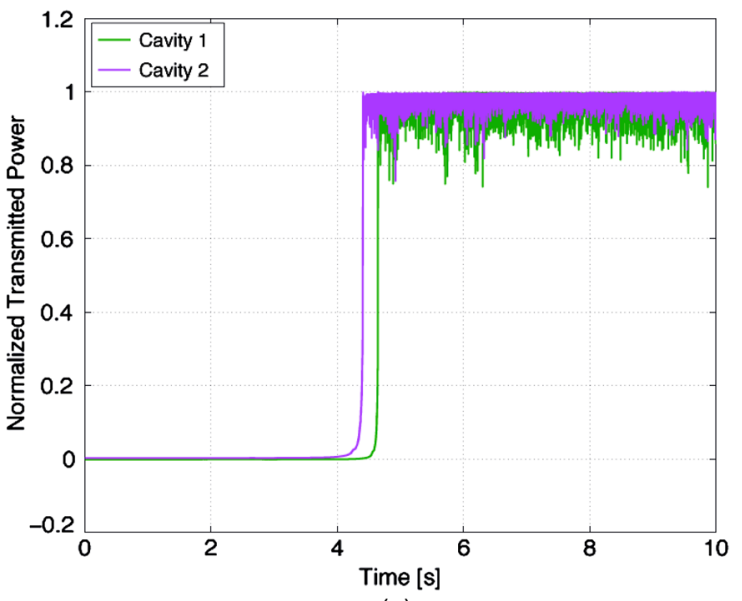

(a)

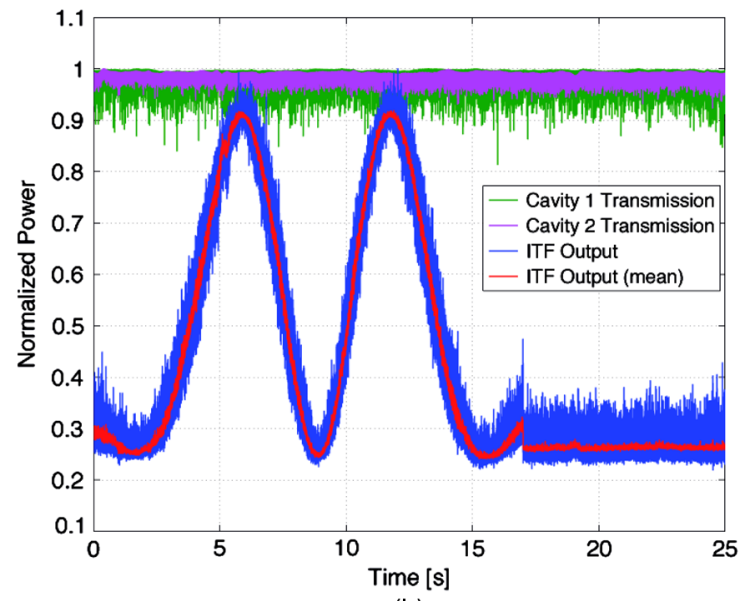

(b)

FIG. 7 (color online). (a) Power transmitted by the two cavities during a typical lock acquisition using the $\mathrm{LG}_{33}$. (b) Evolution of power on the interferometer output, while the cavities are locked, when the Michelson length is modulated with a PZT actuator. At $t=17 \mathrm{~s}$ a correction signal is applied and the Michelson is then locked on the dark fringe. 


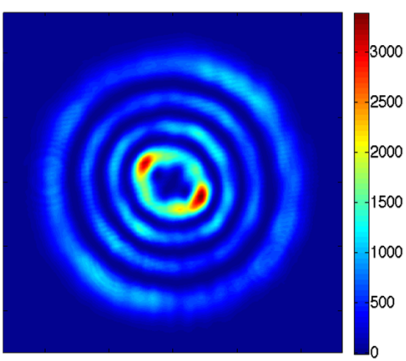

(a)

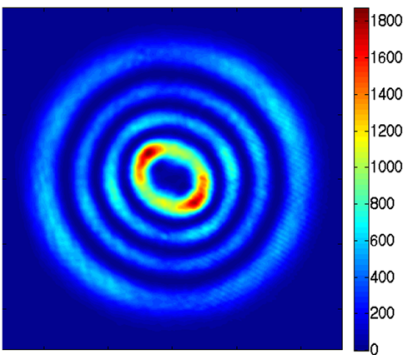

(c)

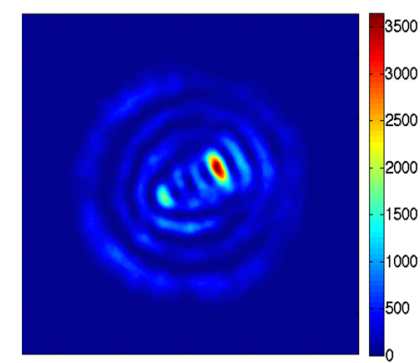

(b)

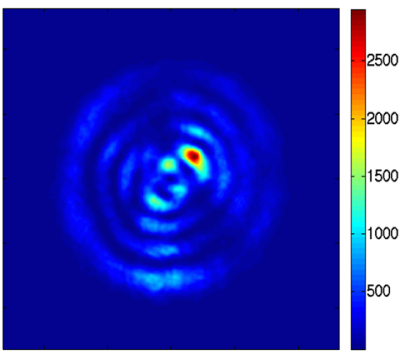

(d)
FIG. 8 (color online). $\quad \mathrm{LG}_{33}$ Transverse intensity distributions measured at (a) cavity 1 transmission, (b) cavity 1 reflection, (c) cavity 2 transmission and (d) cavity 2 reflection.

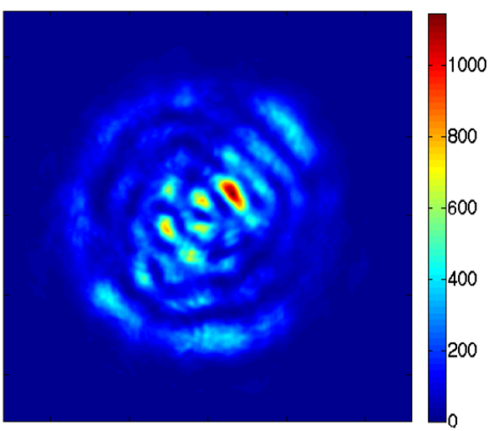

FIG. 9 (color online). Transverse power distribution at the interferometer output when the cavities are resonant and the Michelson is set on the dark fringe condition.

$$
V=\frac{P_{\max }-P_{\min }}{P_{\max }+P_{\min }},
$$

where $P_{\max }$ and $P_{\min }$ are the maximum and minimum power levels measured at the interferometer output. The maximum visibility obtained using the Gaussian beam has been $96 \%$, while the maximum visibility achieved using a $\mathrm{LG}_{33}$ beam has been $84 \%$. In conclusion, a significant degradation of the maximum visibility is observed when passing from the Gaussian to the $\mathrm{LG}_{33}$ beam.

\section{INTERFEROMETER SIMULATION AND COMPARISON WITH THE DATA}

To explain the obtained results, we have performed a numerical simulation of the experiment using the OSCAR tool [22,23]. OSCAR is a Matlab optical fast fourier transform based simulation package. It allows one to propagate an arbitrary beam shape in a complex optical system considering several imperfections: mirror surface quality defects, mode mismatching, mirror misalignments and clipping by optical apertures. The OSCAR package has been widely tested and used for several years in the gravitational-wave community.

We have tuned the simulation parameters using the experimental data in the following way:

(1) Input beam. - The beam transmitted by the mode cleaner has been simulated by propagating a perfect Gaussian beam through the theoretical pattern of the phase plate and then through a simulated modecleaner cavity. The simulated output beam shows a modal purity equal to the measured one (99\%).

(2) Mirror defects.- The transmitted beams are not influenced by small misalignments and small mismatching, since these imperfections create modes nondegenerate with the $\mathrm{LG}_{33}$ and, hence, nonresonant in the cavity. Only mirror defects are responsible for the degradation of the transmitted beams, as a consequence of the degeneracy of the $\mathrm{LG}_{33}$. The amplitudes of mirror defects are inserted in the simulation by matching the shapes and the overlap integrals of the simulated beams with the measured ones. Only the insertion of the astigmatism has been necessary to reproduce the data. Moreover, since we have observed that only the rotation of the end mirrors around the optical axes changes the shape of the transmitted beam, the astigmatism has been introduced in the simulation only on the end mirrors.

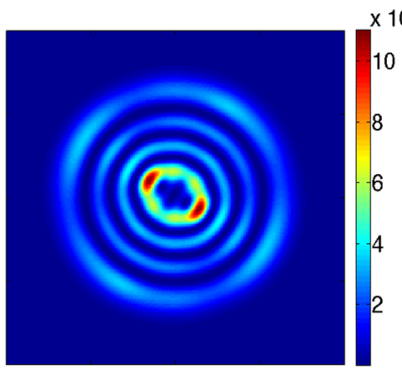

(a)

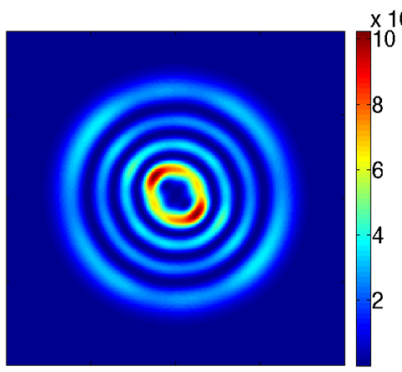

(c)

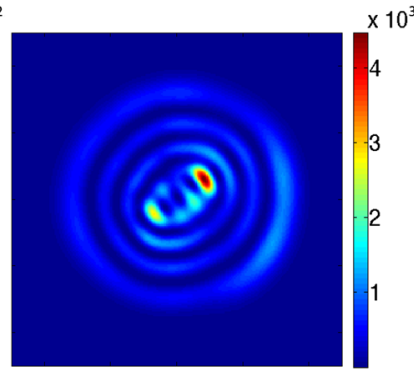

(b)

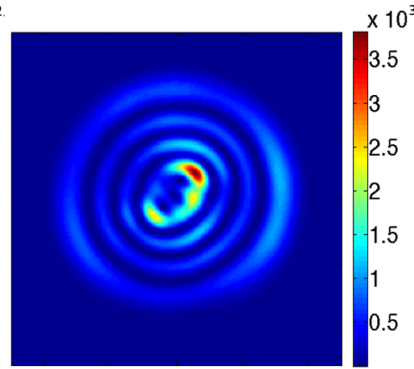

(d)
FIG. 10 (color online). Simulated $\mathrm{LG}_{33}$ intensity distributions at (a) cavity 1 transmission, (b) cavity 1 reflection, (c) cavity 2 transmission and (d) cavity 2 reflection. 
TABLE II. Model parameters.

\begin{tabular}{|c|c|c|c|c|}
\hline & \multicolumn{2}{|c|}{ Astigmatism } & \multicolumn{2}{|c|}{ Misalignments } \\
\hline & Value & Orientation & ITM & ETM \\
\hline Cavity 1 & $0.15 \%$ & $11.65^{\circ}$ & $\begin{array}{c}7 \mu \operatorname{rad}(x) \\
-35 \mu \operatorname{rad}(y)\end{array}$ & $\begin{array}{l}-15 \mu \operatorname{rad}(x) \\
-25 \mu \operatorname{rad}(y)\end{array}$ \\
\hline Cavity 2 & $0.11 \%$ & $0.4^{\circ}$ & $\begin{array}{c}9 \mu \operatorname{rad}(x) \\
-38 \mu \operatorname{rad}(y)\end{array}$ & $\begin{array}{c}25 \mu \operatorname{rad}(x) \\
-39 \mu \operatorname{rad}(y)\end{array}$ \\
\hline
\end{tabular}

The results are shown in Figs. 10(a)-10(c). The astigmatism introduced $(\sim 0.1 \%-0.15 \%)$ is compatible with the surface quality of the used mirrors.

(3) Mismatching.-The mismatching between the input mode and the cavity mode has been inserted in the simulation by tuning the amplitude of modes $\mathrm{LG}_{23}$ and $\mathrm{LG}_{43}$ with the measured one (Fig. 5). This has been obtained inserting a pure waist position error of $1.25 \mathrm{~cm}$. The alternative choice of inserting a pure waist size error gives similar results.

(4) Misalignments.-The values of mirror misalignments are inserted in the simulation by matching the shapes and the overlap integrals of the simulated reflected beams with the measured ones. The simulated reflected shapes are shown in Figs. 10(b)-10(d). The astigmatism values inserted in the simulation are the ones found in point (2). Once the shapes and overlap integrals for the reflected beams are matched, we have checked that the simulated and observed cavity scans are in agreement (see Fig. 5 as well as Fig. 12 below).

The simulation parameters obtained are reported in Table II.

After this parameter tuning, two cross-checks have been performed:

(a) The dark fringe image and fringe visibility have been simulated without any further tuning of the simulation. The simulated image is shown in Fig. 11, and it is compared with the measured one. The simulated fringe visibility $(83 \%)$ is consistent with the measured one $(84 \%)$ and the two images are similar.

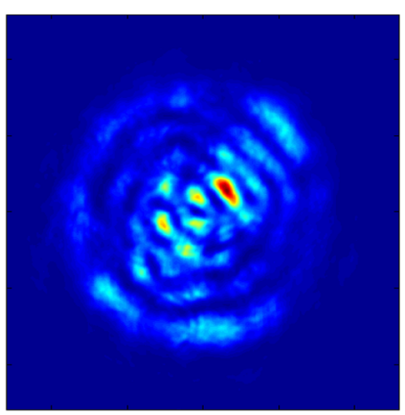

(a)

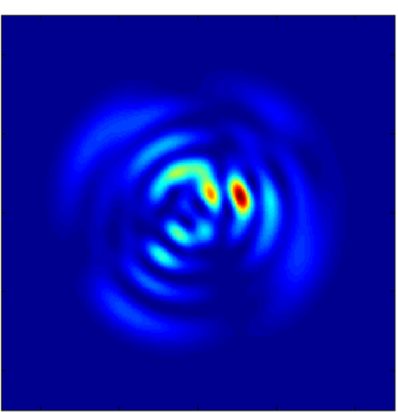

(b)
FIG. 11 (color online). $\quad \mathrm{LG}_{33}$ dark fringe intensity distributions. (a) Measured (already shown in Fig. 9) and (b) simulated.

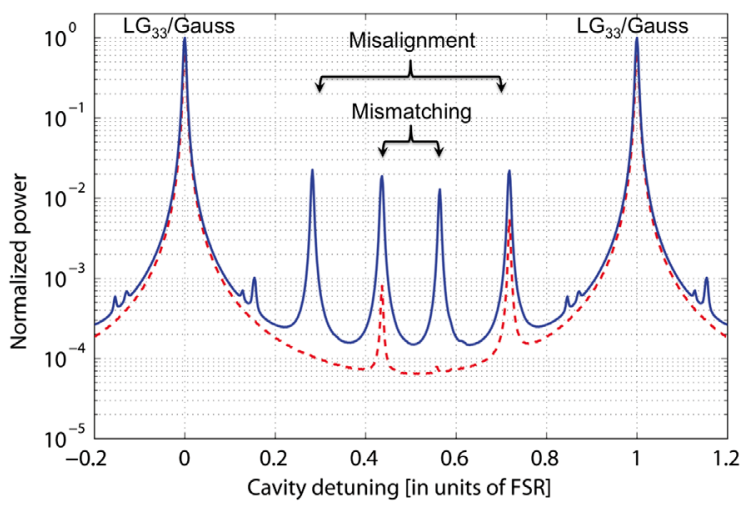

FIG. 12 (color online). Simulated resonance scans for Gaussian (red dotted curve) and $\mathrm{LG}_{33}$ (blue solid curve) beams obtained using the model parameters reported in Table II. The main resonance peaks of the Gaussian and $\mathrm{LG}_{33}$ modes have been superposed in order to highlight their different sensitivities to misalignments and mismatching.

(b) A simulation was performed with the Gaussian beam using the same parameters found in points (2), (3), (4) and reported in Table II. The obtained results (cavity scans and interferometer visibility) are in agreement with the observed values, suggesting that the simulation tuning made for the $\mathrm{LG}_{33}$ also works for the Gaussian mode. The simulated interferometer visibility is $98 \%$, consistent with the measured one (96\%). The simulated cavity scans for the Gaussian beams and $\mathrm{LG}_{33}$ are shown in Fig. 12.

The agreement between simulations and measurements indicates that we are able to explain the images and interferometer visibility obtained with the $\mathrm{LG}_{33}$ in terms of the astigmatism of the cavity mirrors, misalignments and mismatching.

\section{A. Impact of the astigmatism and misalignments/mismatching}

To understand the relative role of astigmatism, misalignments and mismatching on the interferometer visibility, the contributions have been separately simulated. In Fig. 13(a)

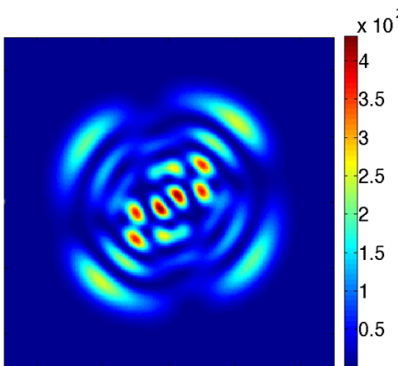

(a)

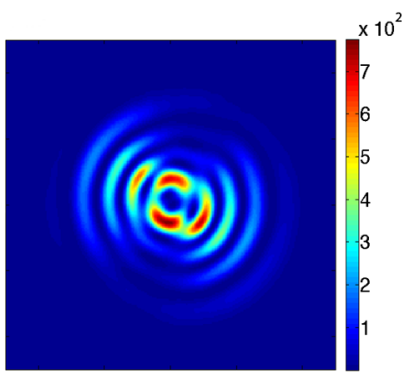

(b)
FIG. 13 (color online). Simulated interferometer output images obtained for (a) astigmatic cavities perfectly aligned and matched; (b) perfect cavities with misalignment and mismatching. 


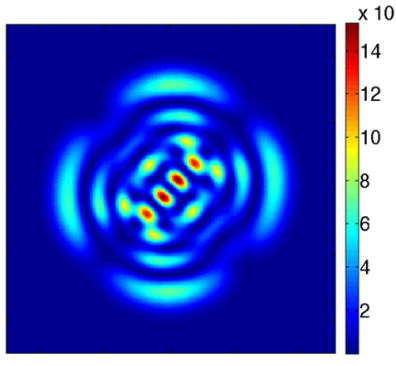

(a)

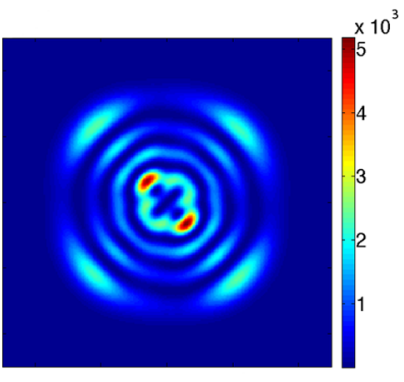

(b)
FIG. 14 (color online). Simulated interferometer output image for the astigmatism axis (a) perfectly aligned and (b) completely misaligned. Note that when the two axes are aligned, the power is more than 1 order of magnitude lower than in the misaligned case.

the interferometer output dark fringe image obtained with two astigmatic arm cavities perfectly aligned and matched is shown, while in Fig. 13(b) the image obtained with two perfect cavities with the misalignments of Table II is shown. In the first case the visibility is $91.4 \%$, while in the latter it is $89 \%$. The interferometer visibility measured is then limited for roughly the same amount by both internal defects and misalignments/mismatching.

\section{B. Impact of the astigmatism axis orientation}

The impact of the relative orientation between the astigmatism axes of the two arm cavities is highlighted in Fig. 14. The interferometer output dark fringe image is shown for the two extreme cases of a perfect alignment (a) or a $90^{\circ}$ misalignment (b) of the astigmatism axis. In the first case, the visibility of the dark fringe is $96.8 \%$, while for the latter it is $9 \%$. To highlight the effect of the astigmatism, in both cases no misalignments or mismatching have been introduced.

\section{CONCLUSIONS}

To demonstrate the possible use of higher-order Laguerre-Gauss modes in future kilometric gravitationalwave detectors, we have operated for the first time a tabletop Fabry-Pérot-Michelson interferometer using a $\mathrm{LG}_{33}$ as input mode. Even though the optical configuration used is simpler with respect to the ones which will probably be used in future gravitational-wave interferometers, it represents an increase in complexity with respect to previous tests with a simple Fabry-Pérot cavity or a simple Michelson interferometer.

The main results obtained are detailed in the following.

With respect to the work described in [15], the robustness of the $\mathrm{LG}_{33}$ generator has been improved, allowing the delivery of an extremely stable beam. The transmitted beam has a quality of $\sim 99 \%$, comparable with the one of the Gaussian beam.
We have shown that it is possible to maintain the quality of the $\mathrm{LG}_{33}$ beam from the generator to the interferometer, through the injection system optics.

The procedure to prealign the interferometer using a Gaussian beam (also used for the alignment of the $\mathrm{LG}_{33}$ generator), was very efficient to obtain a fast and quasioptimal alignment of the interferometer. The mode matching of the input beam with the Fabry-Pérot cavities was also made using the Gaussian beam, obtaining a very high coupling (higher than 96\%) for the $\mathrm{LG}_{33}$.

The interferometer was locked robustly using the $\mathrm{LG}_{33}$, with the two cavities resonant with the laser light and the Michelson set on the dark fringe condition.

Finally, we have demonstrated the degradation of the interferometer contrast of the $\mathrm{LG}_{33}$ with respect to the Gaussian beam due to the degeneracy problem. The contrast degradation observed ( $84 \%$, for $\mathrm{LG}_{33}$ with respect to $96 \%$ obtained with the Gaussian beam), as well as the interferometer optical properties have been explained using a numerical simulation based on the OSCAR package.

\section{NEXT STEPS}

So far, no fundamental obstacles have been observed for the operation of $\mathrm{LG}_{33}$ in future $\mathrm{GW}$ detectors, except the crucial problem of the degradation of the beam quality (and then of the interferometer contrast), due to the degeneracy of LG modes with other modes of the same order. To achieve mode purities compatible with GW detector requirements, mirror surface qualities much higher than the available ones will be necessary $[18,19]$. For these reasons, this technique does not seem mature for short-term upgrades of the GW detectors.

Even if polishing and coating techniques have shown good progress in past years, it is difficult to predict their improvements in the next decade. However, the effect of $\mathrm{LG}_{33}$ degeneracy can in principle be mitigated also with in situ thermal correction techniques of a mirror surface figure $[24,25]$. These techniques, when experimentally demonstrated, could relax the requirements on mirror surface quality and make possible the use of LG modes on a longer time scale.

To this aim, the table-top interferometer described in this work will be mainly used in the near future to test in situ thermal correction techniques.

We are also considering an increase to the complexity of the table-top interferometer, adding a power and/or signal recycling mirror, and thus checking optical performance of $\mathrm{LG}_{33}$ in a configuration closer to the one used for advanced gravitational-wave interferometers.

\section{ACKNOWLEDGMENTS}

We would like to thank J. Degallaix for the assistance with OSCAR; J. Degallaix, M. Granata and G. Vajente for the useful discussions; $\mathrm{B}$. Whiting for the reading of the 
manuscript; the Laboratoire d'Annecy-le-Vieux de Physique des Particules for the support with the digital control system and photodiode readout; H. Halloin and P. Prat for discussions and support with the analog electronics. M. Granata and R. Ward have contributed to the realization of the $\mathrm{LG}_{33}$ generator described in [15]. This work is supported by the Agence Nationale de la Recherche under Grant No. 2010-BLANC-0416-01. M. T. is also supported by the European Gravitational Observatory.
[1] H. Grote (for LIGO Scientific Collaboration), Classical Quantum Gravity 27, 084003 (2010).

[2] B. P. Abbott et al. (LIGO Scientific Collaboration), Rep. Prog. Phys. 72, 076901 (2009).

[3] F. Acernese et al. (Virgo Collaboration), Classical Quantum Gravity 25, 184001 (2008).

[4] J. Abadie et al., Classical Quantum Gravity 27, 173001 (2010)

[5] B. Wilke et al., Classical Quantum Gravity 23, S207 (2006).

[6] Y. Aso, Y. Michimura, K. Somiya, M. Ando, O. Miyakawa, T. Sekiguchi, D. Tatsumi, and H. Yamamoto, Phys. Rev. D 88, 043007 (2013).

[7] G. Harry (for LIGO Scientific Collaboration), Classical Quantum Gravity 27, 084006 (2010).

[8] Virgo Collaboration, arXiv:1408.3978.

[9] E. D’Ambrosio, Phys. Rev. D 67, 102004 (2003).

[10] B. Mours, E. Tournefier, and J.-Y. Vinet, Classical Quantum Gravity 23, 5777 (2006).

[11] J.-Y. Vinet, Living Rev. Relativity 12, 5 (2009).

[12] H. Kogelnik and T. Li, Appl. Opt. 5, 1550 (1966).

[13] A. E. Siegman, Lasers (University Science Books, Sausalito, CA, 1986).

[14] P. Fulda, K. Kokeyama, S. Chelkowski, and A. Freise, Phys. Rev. D 82, 012002 (2010).
[15] M. Granata, C. Buy, R. Ward, and M. Barsuglia, Phys. Rev. Lett. 105, 231102 (2010).

[16] L. Carbone, C. Bogan, P. Fulda, A. Freise, and B. Willke, Phys. Rev. Lett. 110, 251101 (2013).

[17] B. Sorazu, P. J. Fulda, B. W. Barr, A. S. Bell, C. Bond, L. Carbone, A. Freise, S. Hild, S. H. Huttner, J. Macarthur, and K. A. Strain, Classical Quantum Gravity 30, 035004 (2013).

[18] T. Hong, J. Miller, H. Yamamoto, Y. Chen, and R. Adhikari, Phys. Rev. D 84, 102001 (2011).

[19] C. Bond, P. Fulda, L. Carbone, K. Kokeyama, and A. Freise, Phys. Rev. D 84, 102002 (2011).

[20] M. Galimberti et al., Presentation at the Einstein Telescope meeting, Budapest, 2010, Einstein Telescope internal document, https://tds.ego-gw.it/.

[21] F. Acernese et al. (Virgo Collaboration), Astropart. Phys. 20, 617 (2004).

[22] J. Degallaix, J. Phys. Conf. Ser. 228, 012021 (2010).

[23] http://www.mathworks.fr/matlabcentral/fileexchange/20607oscar.

[24] R. A. Day, G. Vajente, M. Kasprzack, and J. Marque, Phys. Rev. D 87, 082003 (2013).

[25] G. Vajente and R. A. Day, Phys. Rev. D 87, 122005 (2013). 